Provided for non-commercial research and education use. Not for reproduction, distribution or commercial use.



This article appeared in a journal published by Elsevier. The attached copy is furnished to the author for internal non-commercial research and education use, including for instruction at the authors institution and sharing with colleagues.

Other uses, including reproduction and distribution, or selling or licensing copies, or posting to personal, institutional or third party websites are prohibited.

In most cases authors are permitted to post their version of the article (e.g. in Word or Tex form) to their personal website or institutional repository. Authors requiring further information regarding Elsevier's archiving and manuscript policies are encouraged to visit:

http://www.elsevier.com/copyright 


\title{
3D features of modified photostructurable glass-ceramic with infrared femtosecond laser pulses
}

\author{
J.M. Fernández-Pradas*, D. Serrano, S. Bosch, J.L. Morenza, P. Serra \\ Universitat de Barcelona, Departament de Física Aplicada i Òptica, Martí i Franquès 1, E-08028 Barcelona, Spain
}

\section{A R T I C L E I N F O}

Article history:

Available online 10 November 2010

Keywords:

Microchannels

Photostructurable glass-ceramic

Femtosecond laser microfabrication

\begin{abstract}
A B S T R A C T
The exclusive ability of laser radiation to be focused inside transparent materials makes lasers a unique tool to process inner parts of them unreachable with other techniques. Hence, laser direct-write can be used to create 3D structures inside bulk materials. Infrared femtosecond lasers are especially indicated for this purpose because a multiphoton process is usually required for absorption and high resolution can be attained. This work studies the modifications produced by $450 \mathrm{fs}$ laser pulses at $1027 \mathrm{~nm}$ wavelength focused inside a photostructurable glass-ceramic $\left(\right.$ Foturan $\left.^{\circledR}\right)$ at different depths. Irradiated samples were submitted to standard thermal treatment and subsequent soaking in HF solution to form the buried microchannels and thus unveil the modified material. The voxel dimensions of modified material depend on the laser pulse energy and the depth at which the laser is focused. Spherical aberration and selffocusing phenomena are required to explain the observed results.
\end{abstract}

@ 2010 Elsevier B.V. All rights reserved.

\section{Introduction}

Femtosecond lasers can produce micrometric size modifications inside transparent materials [1]. Because the energy gap transitions between valence and conduction bands of transparent materials are larger than the photon energy, the femtosecond laser pulses must be tightly focused with high NA lenses to promote non-linear absorption processes such as multiphoton absorption or tunnel ionization [2]. In these cases, the volume where absorption occurs is considerably reduced compared with the linear absorption case. Thus, femtosecond lasers become the best instrument for 3D directwrite fabrication, allowing producing waveguides, optical, MEMS and microfluidic devices integrated inside the same material with a single tool [3].

Foturan $^{\circledR}$ is a photostructurable glass-ceramic first designed for microchannel production through photolithographic processes [4]. Basically, it is a lithium silicate glass doped with cerium ions as photosensitive agent and silver ions for promoting glass crystallization once photosensitized with UV radiation. The transformed crystalline phase is preferentially etched in HF solutions at recommended concentrations in the range $5-10 \%$ in front of the amorphous glass phase. Thus, micrometric channels can be formed on its surface. However, a laser can be used to locally deliver photons able to photosensitize Foturan in substitution of the UV lamp illumination and the photolithographic masks. In this way, patterns

\footnotetext{
* Corresponding author.

E-mail address: jmfernandez@ub.edu (J.M. Fernández-Pradas).
}

can be written not only on the surface but inside the bulk, adding 3D capabilities to the Foturan microfabrication. Excimer and Nd: YAG lasers with nanosecond pulses and UV wavelengths, as well as femtosecond pulsed lasers with infrared wavelengths have been used for Foturan microfabrication [5-10].

In the work presented here, we investigate the formation of microchannels buried at different depths in Foturan slides after irradiation with $450 \mathrm{fs}$ laser pulses of $1027 \mathrm{~nm}$ wavelength. The microchannel dimensions are compared with calculated intensity profile distribution of the focused beam inside Foturan to investigate the mechanisms that could affect the resolution of the technique, especially in the direction of the propagating beam.

\section{Experimental procedures}

Foturan ${ }^{\circledR}$ slides from Mikroglas Chemtech $\mathrm{GmbH}$ with $1 \mathrm{~mm}$ thickness were cut into $1 \mathrm{~cm} \times 1 \mathrm{~cm}$ squares. A femtosecond laser source delivering pulses of $450 \mathrm{fs}$ with central wavelength of $1027 \mathrm{~nm}$ was focused through a $\times 50$ objective with 0.55 NA into the samples at a controlled depth for promoting Foturan photomodification. The laser beam had a Gaussian intensity profile with quality factor $\mathrm{M}^{2}$ of 1.2 . Buried microchannels were prepared by moving samples horizontally while irradiating with the laser, leaving $10 \mu \mathrm{m}$ between consecutive laser pulses. Series of microchannels buried at different depths and at different laser pulse energies were marked in a same sample square. Afterwards, samples were submitted to standard thermal treatment: first, temperature was raised up to $500^{\circ} \mathrm{C}$ with a ramp of $5^{\circ} \mathrm{C} / \mathrm{min}$ and kept at $500^{\circ} \mathrm{C}$ for $1 \mathrm{~h}$; immediately after, it was further increased up to $600^{\circ} \mathrm{C}$ at 


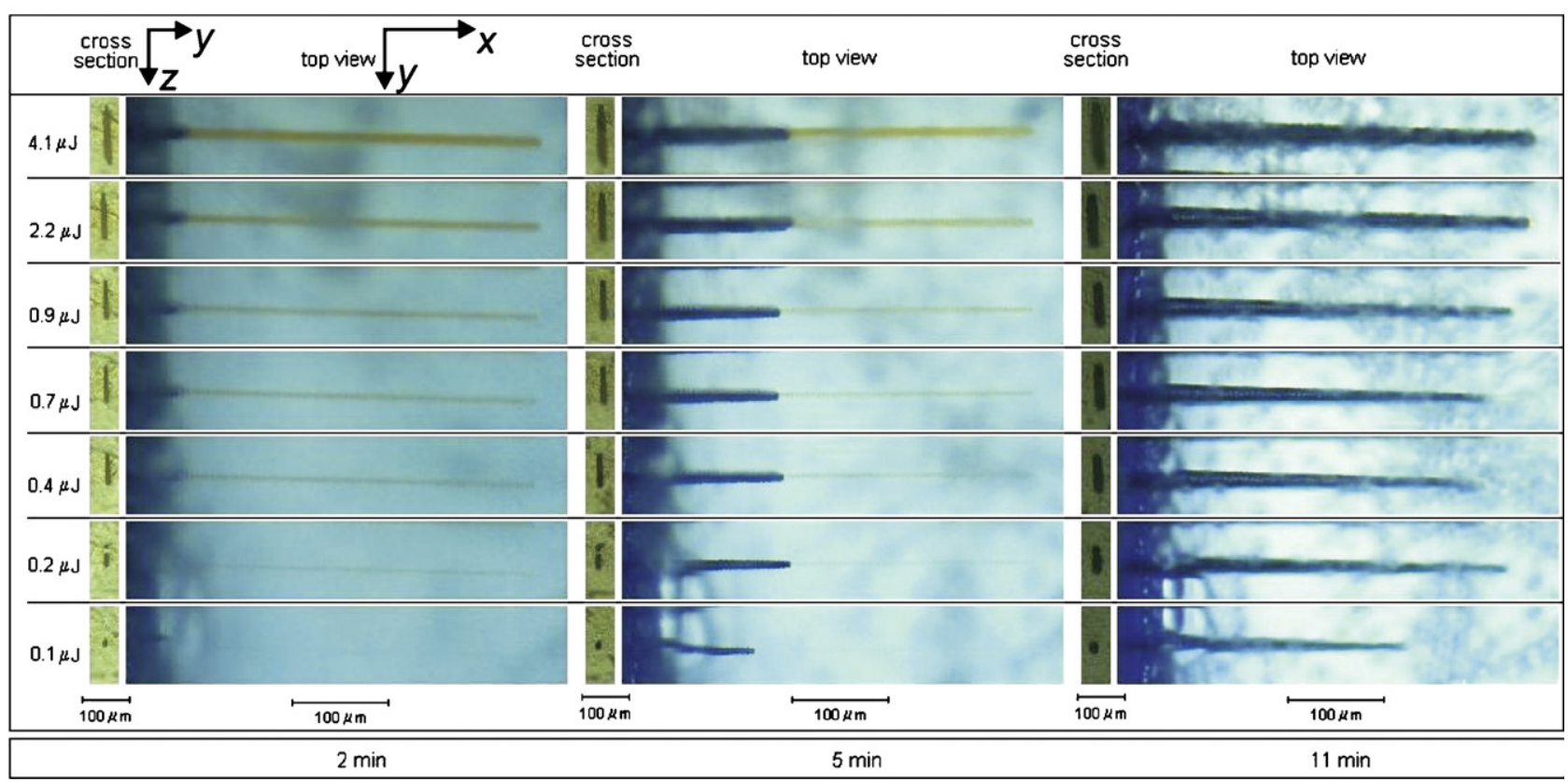

Fig. 1. Cross-section ( $y z$ plane) and top ( $x y$ plane) views of the formation of microchannels fabricated in Foturan at different laser pulse energies. Images were taken after 2 , 5 and $11 \mathrm{~min}$ of sample immersion in $10 \% \mathrm{HF}$.

$3{ }^{\circ} \mathrm{C} / \mathrm{min}$ and held at $600^{\circ} \mathrm{C}$ for another hour; and finally, temperature was decreased down to room temperature at $5^{\circ} \mathrm{C} / \mathrm{min}$. After thermal treatment, samples were cut in a plane perpendicular to the microchannels with a slow speed diamond saw to allow direct exposure to modified material for the final etching step. Selective etching was performed by immersion of the samples in $10 \% \mathrm{HF}$ solution with ultrasonic agitation. In order to study the time evolution, the etching process was interrupted at different times by rinsing samples in distilled water for their inspection at the microscope.

To clarify notation henceforth, the $z$ axis corresponds to the beam propagation direction; the $x$ axis corresponds to the longitudinal direction of the microchannels; and consequently, the $y$ axis corresponds to the direction perpendicular to the $z$ axis in the cross-section plane of the microchannels (Fig. 1).

\section{Results}

Optical microscopy inspection of microchannel formation reveals that chemical etching advances along the microchannel ( $x$ direction) at similar speed for most of the irradiation laser pulse energies (Fig. 1). Microchannels cross-section is elongated along the laser beam direction ( $z$ direction), with this dimension increasing with the laser pulse energy used during irradiation. Measurement of microchannels length at three different etching times allowed to determine the etch rate for the different pulse energy conditions (Fig. 2). Only the microchannel irradiated with $0.1 \mu \mathrm{J}$ pulses presents an etch rate below $30 \mu \mathrm{m} / \mathrm{min}$. For the rest of conditions, the etch rate slowly increases with laser pulse energy from 35 to $41 \mu \mathrm{m} / \mathrm{min}$. The described cross-section shape rapidly appears after the first seconds under immersion in the $10 \% \mathrm{HF}$ solution, and it continues growing by etching the microchannel wall at an etch rate of about $0.7 \mu \mathrm{m} / \mathrm{min}$ which is similar to that found for non-irradiated material [10]. The corresponding etch rate ratios of modified Foturan versus non-irradiated Foturan can be then calculated (Fig. 2). Concomitantly with the etch rate, their values slowly increase with laser pulse energy above $0.2 \mu \mathrm{J}$, reaching maximum etch rate ratios of 58, similar to those obtained in the fabrication of microchannels in the laser incidence direction [10].
Fig. 3 shows the cross-section view of a sample with series of microchannels formed at different focusing depths with different pulse energies. When laser pulse energy is above $0.4 \mu \mathrm{J}$, the $z$ length of the microchannels cross-section increases with the depth at which the microchannel is buried with respect to the first surface that the laser beam is crossing. However, at lower energies, this length remains nearly constant or is sometimes reduced with increasing depth down to the limit that microchannels were not developed at a certain depth in the lowest pulse energy series. Because cross-section dimensions were continuously growing during the etching process, we extrapolated their values at $t=0$, and assumed that they corresponded to the voxel dimensions of the laser modified material. Voxel sizes as small as 2 and $10 \mu \mathrm{m}$ in the $y$ and $z$ directions, respectively, were found. Plot of the $z$-length of modified material ( $\left.L_{Z 0}\right)$ vs depth (Fig. 4 ) shows an exponential relationship for microchannels made with the same pulse energy, except for those made with the lowest pulse energies ( 0.21 and $0.16 \mu \mathrm{J})$. Moreover, $L_{z 0}$ increases logarithmically with increasing pulse energy (Fig. 5).

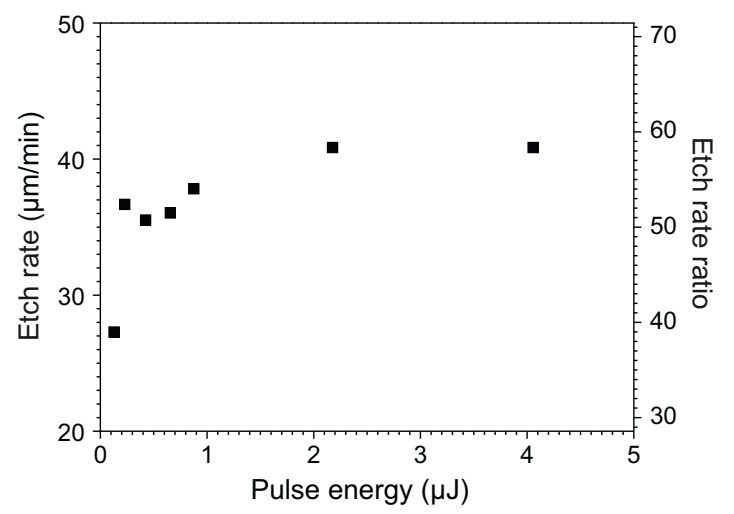

Fig. 2. Plot of etch rate and etch rate ratio vs laser pulse energy of microchannels buried in Foturan. 


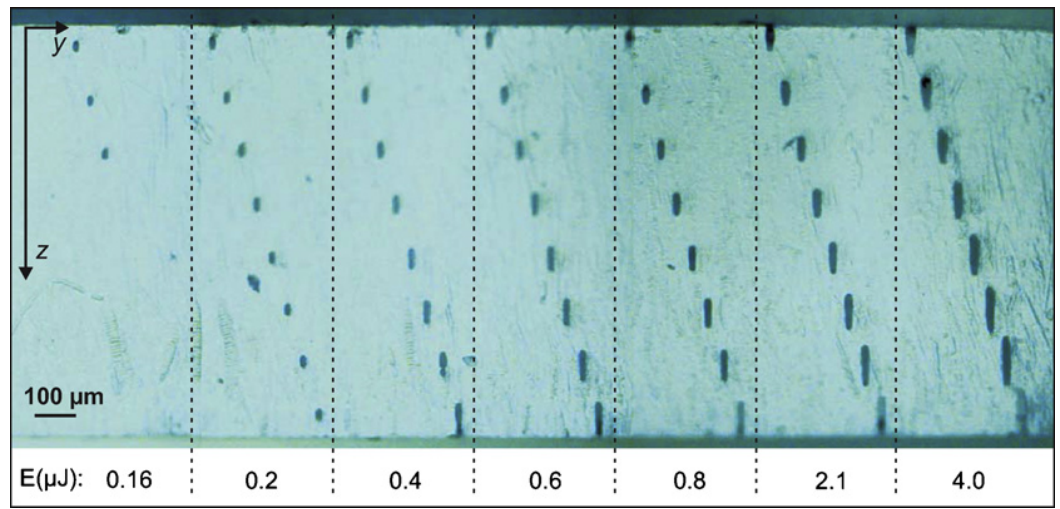

Fig. 3. Optical micrograph of the cross-section of a Foturan sample with microchannels made at different depths and pulse energies after 9 min etching. Laser beam propagates in the $z$ direction.

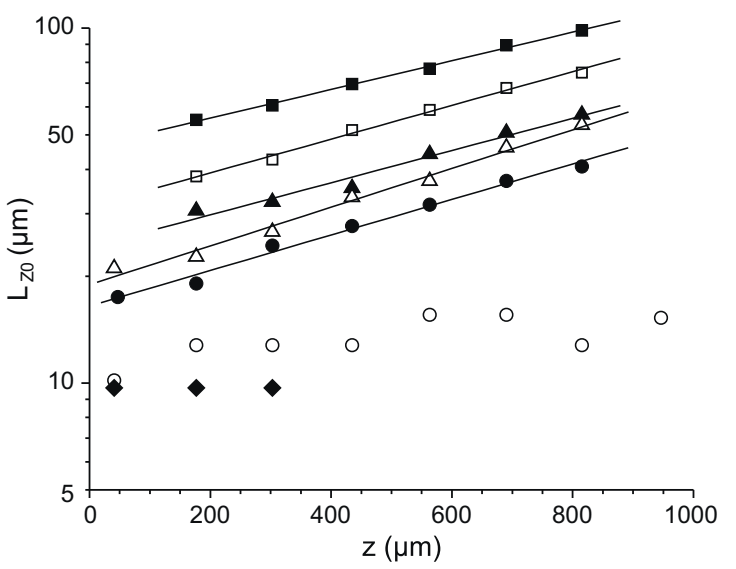

Fig. 4. Plot of the $z$-length of the voxel $v s$ depth at different laser pulse energies: ( $0.16,(\bigcirc) 0.2,(\bullet) 0.4,(\triangle) 0.6,(\Delta) 0.8,(\square) 2.1$, and $(\square) 4.0 \mu \mathrm{J}$.

\section{Discussion}

Results show that the cross-section shape of the microchannel, especially its $z$-length, changes depending on the depth at which it is buried. Because the observed differences must be related to the laser voxel shape, it becomes clear that the intensity distribution of the focused beam suffers some distortion when propagating through the Foturan sample. It is an unavoidable fact that a refrac-

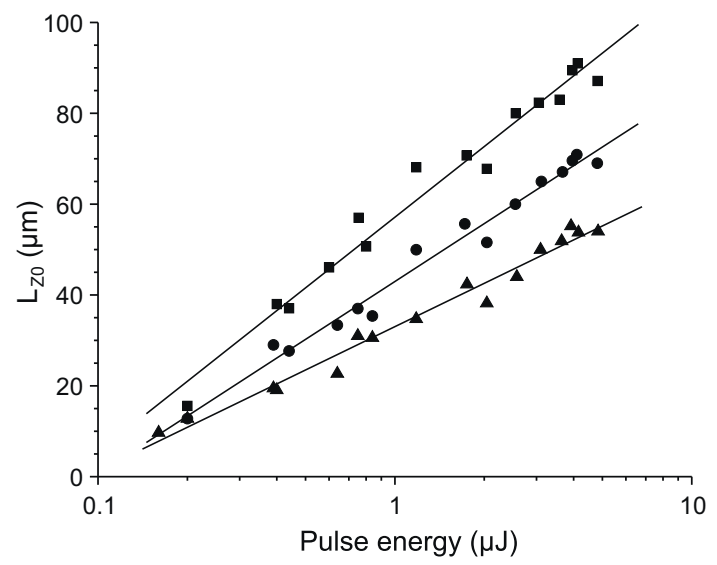

Fig. 5. Plot of the $z$-length of the voxel $v s$ laser pulse energy at different depths: ( $\mathbf{\Delta})$ $180,(\bullet) 435$, and (ם) $690 \mu \mathrm{m}$. tive indices mismatch induces spherical aberration. Therefore, this is the first effect that we have considered. For this, the intensities inside the sample were computed on the basis of the scalar theory of diffraction [11], which is good enough approximation at the focusing conditions of this work. We assumed that a Gaussian beam, defined by its waist radius, impinged normally from air into Foturan. According to the depth where the laser was focused, the Gaussian beam had a certain beam radius and wavefront curvature at the interface. Because of refraction at the interface, the beam curvature changed and, besides, acquired spherical aberration [12]. Then, the aberrated beam was propagated from the interface by means of standard computational procedures for scalar diffraction and the isophotes were computed near the focusing regions. Details about the computation procedure can be found in Ref [13]. Fig. 6 shows the obtained intensity profiles along the beam axis calculated for 450 fs laser pulses of $0.2 \mu \mathrm{J}$, focused into Foturan at depths corresponding to the experimental conditions of the sample presented in Fig. 3. An example of isophote distribution in the $y z$ plane is shown in insert of Fig. 6. As it can be seen, spherical aberration produces distortion of the beam intensity distribution promoting an enlargement along the propagation beam axis, $z$, together with a decreasing of the maximum peak value with the increasing focus-

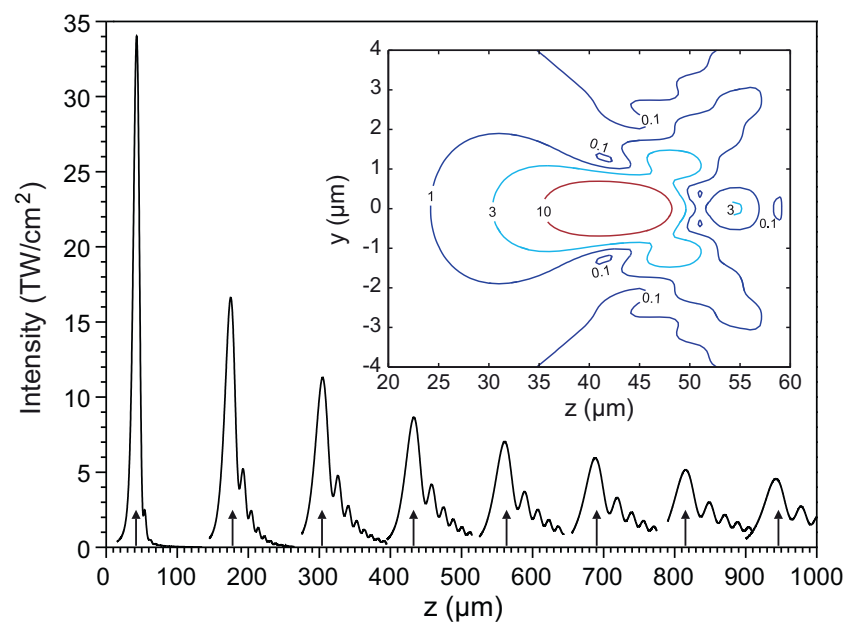

Fig. 6. Calculated laser beam intensity profile along the beam axis, $z$, of $450 \mathrm{fs}, 0.2 \mu \mathrm{J}$ pulses with Gaussian intensity distribution focused at different depths with $0.55 \mathrm{NA}$ objective inside a medium with 1.51 refractive index. Arrows indicate the depth at which the microchannels appear in Figure 3. Insert shows calculated isophotes distribution in the $y z$ plane for the lowest focusing depth. Numbers in the insert indicate the corresponding intensity of the isophote in $\mathrm{TW} / \mathrm{cm}^{2}$. 
ing depth. The intensity distribution when using different pulse energies is basically the same except for a proportional multiplying factor. The depth at which the calculated maxima appear corresponds quite well with the position where microchannels were formed (Fig. 6). However, it has not been possible to identify a unique intensity threshold value that matches the measured size of all the photosensitized cross-section areas. Our simulations show that spherical aberration produces stronger intensity distribution enlargement and stronger peak decrease than required to fit the experimental results. Laser beam deviations from the Gaussian profile may contribute to these differences. Moreover, self-focusing can be also involved in counteracting the intensity dispersion produced by spherical aberration. Self-focusing is a non-linear process that takes into account the refractive index dependence with light intensity and that often appears when working with femtosecond laser pulses [14-19]. Above a critical power value, the Gaussian intensity profile of the beam induces a refractive-index variation within the material that acts as a converging lens that promotes additional focusing. At $1027 \mathrm{~nm}$ wavelength the critical power for self-focusing in Foturan can be estimated in between 1.3 and 2.6 MW [16-19]. Thus, for pulse energies above $0.6 \mu \mathrm{J}$ this mechanism may compensate for the spherical aberration one, allowing the photomodification of material at depths and energies that otherwise should not occur.

\section{Conclusions}

Microchannels can be fabricated in Foturan slides buried at different depths with femtosecond laser pulses at $1027 \mathrm{~nm}$ wavelength. Microchannel cross-section is elongated along the laser beam direction. The microchannel size along this direction can be controlled through adjustment of the pulse energy for the different depths. The voxel size in this direction follows a logarithmic relationship with pulse energy, and an exponential relationship with focusing depth. However, real microchannel cross-section sizes will always be larger than voxel sizes because continuous wall etching during microchannel formation. Computation of the propagation of a Gaussian beam taking into account the spherical aberration mechanism occurring at the air-Foturan surface gives good agreement with the positions at which microchannels are formed. However, self-focusing should also be considered as a counteracting mechanism in order to explain the smaller real voxel size and the presence of some microchannels at large focusing depths that should not be formed if only spherical aberration is taken into account.

\section{Acknowledgements}

This work is part of a research program funded by MCI of the Spanish Government (Projects MAT2007-62357 and CSD200800023), and Fondo Europeo de Desarrollo Regional (FEDER).

\section{References}

[1] R.R. Gattass, E. Mazur, Femtosecond laser micromachining in transparent materials, Nat. Photonics 2 (2008) 219-225.

[2] C.B. Schaffer, A. Brodeur, E. Mazur, Laser-induced breakdown and damage in bulk transparent materials induced by tightly focused femtosecond laser pulses, Meas. Sci. Technol. 12 (2001) 1784-1794.

[3] K. Sugioka, Y. Hanada, K. Midorikawa, Three-dimensional femtosecond laser micromachining of photosensitive glass for biomicrochips, Laser Photon. Rev. 4 (2010) 386-400.

[4] T.R. Dietrich, W. Ehrfeld, M. Lacher, M. Krämer, B. Speit, Fabrication technologies for microsystems utilizing photoetchable glass, Microelectron. Eng. 30 (1996) 497-504.

[5] P.D. Fuqua, D.P. Taylor, H. Helvajian, W.W. Hansen, M.H. Abraham, A UV directwrite approach for formation of embedded structures in photostructurable glass-ceramics, Mat. Res. Soc. Symp. Proc. 624 (2000) 79-86.

[6] S. Juodkazis, K. Yamasaki, V. Mizeikis, S. Matsuo, H. Misawa, Formation of embedded patterns in glasses using femtosecond irradiation, Appl. Phys. A 79 (2004) 1549-1553.

[7] J. Kim, H. Berberoglu, X. Xu, Fabrication of microstructures in photoetchable glass-ceramics using excimer and femtosecond lasers, J. Microlith. Microfab. Microsyst. 3 (2004) 478-485.

[8] T. Hongo, K. Sugioka, H. Niino, Y. Cheng, M. Masuda, I. Miyamoto, H. Takai, K. Midorikawa, Investigation of photoreaction mechanism of photosensitive glass by femtosecond laser, J Appl. Phys. 97 (2005), 063517 1-4.

[9] K. Sugioka, Y. Cheng, K. Midorikawa, Three-dimensional micromachining of glass using femtosecond laser for lab-on-a-chip device manufacture, Appl Phys. A 81 (2005) 1-10.

[10] J.M. Fernández-Pradas, D. Serrano, P. Serra, J.L. Morenza, Laser fabricated microchannels inside photostructurable glass-ceramic, Appl. Surf. Sci. 255 (2009) 5499-5502.

11] J.W. Goodman, Introduction to Fourier Optics, McGraw-Hill, New York, 1968

[12] V.N. Mahajan, Optical Imaging and Aberrations, Part II, SPIE Press, Bellingham, 1998 (Chapter 1)

[13] S. Bosch, J. Ferré-Borrull, Analysis of waves near focus: method and experimental test, Appl. Phys. Lett. 80 (2002) 1686-1688.

[14] R.W. Boyd, Nonlinear Optics, third ed., Academic Press, Oxford, 2008.

[15] Y. Shimotsuma, K. Hirao, J. Qiu, K. Miura, Nanofabrication in transparent materials with a femtosecond pulse laser, J. Non-Cryst. Solids 352 (2006) 646-656.

[16] B. Fisette, F. Busque, J.-Y. Degorce, M. Meunier, Three-dimensional crystallization inside photosensitive glasses by focused femtosecond laser, Appl. Phys. Lett. 88 (2006) 91104

[17] M. Oujja, S. Pérez, E. Fadeeva, J. Koch, B.N. Chichkov, M. Castillejo, Three dimensional microstructuring of biopolymers by femtosecond laser irradiation, Appl. Phys. Lett. 95 (2009) 263703.

[18] E.G. Gamaly, S. Juodkazis, K. Nishimura, H. Misawa, B. Luther-Davies, L. Hallo P. Nicolai, V.T. Tikhonchuk, Laser-matter interaction in the bulk of a transparent solid: confined microexplosion and void formation, Phys. Rev. B 73 (2006) 214101.

[19] S. Juodkazis, V. Mizeikis, S. Matsuo, K. Ueno, H. Misawa, Bull. Chem. Soc. Jpn. 4 (2008) 411-448. 